PROCEEDINGS OF THE AMERICAN MATHEMATICAL SOCIETY

Volume 127, Number 4, April 1999, Pages 1109-1117

S 0002-9939(99)04618-3

\title{
LOEWNER'S THEOREM FOR KERNELS HAVING A FINITE NUMBER OF NEGATIVE SQUARES
}

\author{
D. ALPAY AND J. ROVNYAK
}

(Communicated by Theodore W. Gamelin)

\begin{abstract}
By a theorem of Loewner, a continuously differentiable real-valued function on a real interval whose difference quotient is a nonnegative kernel is the restriction of a holomorphic function which has nonnegative imaginary part in the upper half-plane and is holomorphic across the interval. An analogous result is obtained when the difference-quotient kernel has a finite number of negative squares.
\end{abstract}

\section{Classical theorem of Loewner}

In this paper we prove a version of Loewner's theorem for classes of meromorphic functions that generalize the Pick-Nevanlinna and Schur classes by a method that reduces the result to the classical case. In the present section the classical version of Loewner's theorem will be stated in two equivalent forms, for the unit circle and for the real line. Background results on reproducing kernel Pontryagin spaces and the generalized Schur and Nevanlinna classes $\mathbf{S}_{\kappa}$ and $\mathbf{N}_{\kappa}$ are discussed in $\S 2$. The main results are stated and proved in $\S 3$.

The Pick class or Nevanlinna class is the set $\mathcal{P}$ of holomorphic functions which are defined and have nonnegative imaginary part on the open upper halfplane $\mathbf{C}_{+}$. The Schur class is the set $\mathcal{S}$ of holomorphic functions which are defined and bounded by one on the open unit disk $\mathbf{D}$. The term kernel is understood to mean a Hermitian kernel, that is, a complex-valued function $K(s, t)$ on a product set $\Omega \times \Omega$ such that $K(t, s)=\overline{K(s, t)}$ for all $s, t \in \Omega$. We call a kernel nonnegative if all matrices

$$
\left(K\left(s_{j}, s_{i}\right)\right)_{i, j=1}^{n}, \quad s_{1}, \ldots, s_{n} \in \Omega, \quad n=1,2,3, \ldots,
$$

are nonnegative.

Let $f(x)$ be a real-valued continuously differentiable function on a nonempty open subset $G$ of the real line $\mathbf{R}$. Define a kernel $K(x, y)$ on $G \times G$ by

$$
K(x, y)=\left\{\begin{array}{cc}
\frac{f(x)-f(y)}{x-y}, & x \neq y, \\
f^{\prime}(x), & x=y .
\end{array}\right.
$$

Received by the editors July 25, 1997.

1991 Mathematics Subject Classification. Primary 30E05, 47A57; Secondary 46C20, 47B50.

Key words and phrases. Loewner, Löwner, Pontryagin space, reproducing kernel, negative squares, Pick, Schur, Nevanlinna.

The second author was supported by the National Science Foundation under DMS-9501304.

(C)1999 American Mathematical Society 
Since $f(x)$ is continuously differentiable, (1.2) is a continuous function on $G \times G$. The following assertions are equivalent:

(i) there is a function $\hat{f} \in \mathcal{P}$ which is holomorphic across $G$ and takes only real values on $G$ such that $\hat{f}(x)=f(x)$ for all $x$ in $G$;

(ii) the kernel (1.2) is nonnegative on $G \times G$.

When $G$ is an interval, this is a theorem of Loewner [11]; see Korányi [9] for a proof by an operator method. The extension to open sets is given in Rosenblum and Rovnyak $[12,13]$; a proof in this case based on reproducing kernel methods may be found in Donoghue [7].

There is a corresponding result for functions $S(z)$ defined on a nonempty open subset $U$ of $\partial \mathbf{D}$ such that $|S(z)|=1$ for all $z \in U$. Assume that $S(z)$ is continuously differentiable in the sense that

$$
S^{\prime}(w)=\lim _{\substack{z \rightarrow w \\ z \in U}} \frac{S(z)-S(w)}{z-w}
$$

exists and is a continuous function of $w$ in $U$. The kernel

$$
K_{S}(w, z)= \begin{cases}\frac{1-S(z) \overline{S(w)}}{1-z \bar{w}}, & w \neq 1 / \bar{z} \\ w S^{\prime}(w) \overline{S(w)}, & w=1 / \bar{z}\end{cases}
$$

is then a continuous function of $w, z \in U$. The following assertions are equivalent:

(i) there is a function $\hat{S} \in \mathcal{S}$ which is holomorphic across $U$ and takes only values of modulus one on $U$ such that $\hat{S}(z)=S(z)$ for all $z$ in $U$;

(ii) the kernel (1.3) is nonnegative on $U \times U$.

For assume (i). Since $\hat{S} \in \mathcal{S}$, the kernel $[1-\hat{S}(z) \overline{\hat{S}(w)}] /(1-z \bar{w})$ is nonnegative on $\mathbf{D} \times \mathbf{D}$, and (ii) follows on extending this to $U \times U$ by continuity. Conversely, assume (ii). Choose an open set $U_{0} \subseteq U$ such that the set $\left\{S(w): w \in U_{0}\right\}$ omits some point of $\partial \mathbf{D}$, say $\gamma$ (a function satisfying (ii) may assume every value of unit modulus infinitely many times on $U$ ). Then

$$
f(x)=i \frac{\gamma+S(\beta(x))}{\gamma-S(\beta(x))}, \quad \beta(x)=\frac{x-i}{x+i},
$$

defines a real-valued continuously differentiable function on an open subset $G_{0}$ of the real line. Applying Loewner's theorem to this function and then transforming back to the disk (cf. (2.1)-(2.3)), we obtain a function $\hat{S} \in \mathcal{S}$ which is holomorphic across $U_{0}$ and takes only values of modulus one on $U_{0}$ such that $\hat{S}(z)=S(z)$ for all $z$ in $U_{0}$. Since any point of $U$ belongs to some such set $U_{0}$, (i) follows.

\section{Reproducing kernel Pontryagin spaces AND the Generalized Schur and NevanlinNa Classes}

The main results on reproducing kernel Pontryagin spaces are due to Schwartz [14] and Sorjonen [15]. They generalize the classical Hilbert space theory as given, for example, in [4]. We follow the recent accounts in $[1,2]$.

A kernel $K(s, t)$ defined on a set $\Omega \times \Omega$ is said to have $\kappa$ negative squares, $\kappa$ a nonnegative integer, if every matrix of the form (1.1) has at most $\kappa$ negative eigenvalues and at least one such matrix has exactly $\kappa$ negative eigenvalues counted according to multiplicity. 
Lemma 2.1. Let $K(s, t)$ be a kernel which has $\kappa$ negative squares on $\Omega \times \Omega$. It is possible to choose $s_{1}, \ldots, s_{n} \in \Omega$ such that the matrix (1.1) is invertible and has $\kappa$ negative eigenvalues.

Proof. Let $s_{1}, \ldots, s_{n} \in \Omega$ be any points such that the matrix $P=\left(K\left(s_{j}, s_{i}\right)\right)_{i, j=1}^{n}$ has $\kappa$ negative eigenvalues. If $P$ has rank $r$, some principal minor of $P$ of order $r$ is nonzero (Dickson [6] gives an elementary proof of this and attributes the result to Kronecker). By rearranging $s_{1}, \ldots, s_{n}$, we can assume that the principal minor for the first $r$ rows and columns is nonvanishing. Partition $P$ so that

$$
P=\left(\begin{array}{ll}
P_{r} & X \\
X^{*} & Y
\end{array}\right)
$$

where $P_{r}=\left(K_{S}\left(s_{j}, s_{i}\right)\right)_{i, j=1}^{r}$. Since $P_{r}$ is invertible,

$$
P=\left(\begin{array}{cc}
1 & 0 \\
X^{*} P_{r}^{-1} & 1
\end{array}\right)\left(\begin{array}{cc}
P_{r} & 0 \\
0 & Y-X^{*} P_{r}^{-1} X
\end{array}\right)\left(\begin{array}{cc}
1 & P_{r}^{-1} X \\
0 & 1
\end{array}\right),
$$

where the 1's indicate identity matrices of appropriate order. Our assumptions imply that rank $P=\operatorname{rank} P_{r}+\operatorname{rank}\left(Y-X^{*} P_{r}^{-1} X\right)$, and so $Y-X^{*} P_{r}^{-1} X=0$. By Sylvester's law of inertia [8, Theorem 4.5.8, p. 223], $P$ and $P_{r}$ have the same number of negative eigenvalues, and so $s_{1}, \ldots, s_{r}$ have the desired property.

Let $\mathfrak{H}$ be a Pontryagin space of functions on a set $\Omega$. A function $K(s, t)$ on $\Omega \times \Omega$ is a reproducing kernel for $\mathfrak{H}$ if for each $s \in \Omega, K(s, \cdot)$ belongs to $\mathfrak{H}$, and

$$
\langle f(\cdot), K(s, \cdot)\rangle_{\mathfrak{H}}=f(s)
$$

for every $f(\cdot)$ in $\mathfrak{H}$. In this case, $K(s, t)$ is a kernel with $\kappa$ negative squares, where $\kappa$ is the negative index of $\mathfrak{H}$. Conversely, every kernel having $\kappa$ negative squares arises in this way. The correspondence between kernels on $\Omega \times \Omega$ having a finite number of negative squares and reproducing kernel Pontryagin spaces of functions on $\Omega$ is one-to-one $[1,2]$.

In many applications, $\Omega$ is a region in the complex plane. Then a kernel $K(w, z)$ on $\Omega \times \Omega$ is called holomorphic if it is holomorphic as a function of $z$ and $\bar{w}$. A kernel having a finite number of negative squares is holomorphic if and only if the associated reproducing kernel Pontryagin space consists of holomorphic functions.

Definition 2.2. Let $\kappa$ be a nonnegative integer.

(1) The generalized Nevanlinna class is the set $\mathbf{N}_{\kappa}$ of functions $f(z)$ which are holomorphic on some subregion $\Omega$ of $\mathbf{C}_{+}$such that the kernel

$$
\frac{f(z)-\overline{f(w)}}{z-\bar{w}}, \quad w, z \in \Omega
$$

has $\kappa$ negative squares.

(2) The generalized Schur class is the set $\mathbf{S}_{\kappa}$ of functions $S(z)$ which are holomorphic on some subregion $\Omega$ of $\mathbf{D}$ such that the kernel

$$
\frac{1-S(z) \overline{S(w)}}{1-z \bar{w}}, \quad w, z \in \Omega
$$

has $\kappa$ negative squares.

By standard interpolation results, $\mathbf{N}_{0}=\mathcal{P}$ and $\mathbf{S}_{0}=\mathcal{S}$ are the classical Pick and Schur classes. Here and in what follows we employ a convention to identify functions with their analytic continuations. This is justified because the number of 
negative squares of a holomorphic kernel is independent of the region on which the kernel is holomorphic [2, Theorem 1.1.4]. We remark that in [1, 2] it is assumed that generalized Schur functions are defined and holomorphic at the origin. This is required in order to construct canonical realizations with the origin as base point. We do not use these realizations here and make no such assumption now.

The classes $\mathbf{N}_{\kappa}$ and $\mathbf{S}_{\kappa}$ are equivalent. For suppose that $f(z)$ and $S(z)$ are holomorphic on subregions of $\mathbf{C}_{+}$and $\mathbf{D}$ and connected by

$$
f(z)=i \frac{1+S(\beta(z))}{1-S(\beta(z))}, \quad \beta(z)=\frac{z-i}{z+i} .
$$

Here we exclude $S(z) \equiv 1$ and assume that $S(z) \neq 1$ on its domain. Then

$$
\frac{f(z)-\overline{f(w)}}{z-\bar{w}}=\varphi(z) \overline{\varphi(w)} \frac{1-S(\beta(z)) \overline{S(\beta(w))}}{1-\beta(z) \overline{\beta(w)}}
$$

where

$$
\varphi(z)=\frac{2 i}{(z+i)[1-S(\beta(z))]} .
$$

Hence $f(z)$ belongs to $\mathbf{N}_{\kappa}$ if and only if $S(z)$ is in $\mathbf{S}_{\kappa}$.

By a theorem of Kreĭn and Langer [10, Theorem 3.2 on p. 382], $\mathbf{S}_{\kappa}$ consists of all functions of the form

$$
S(z)=S_{0}(z) / B(z), \quad B(z)=\prod_{j=1}^{\kappa} \frac{z-c_{j}}{1-z \bar{c}_{j}},
$$

where $S_{0}(z)$ is in $\mathbf{S}_{0}, c_{1}, \ldots, c_{\kappa}$ are points in $\mathbf{D}$ (not necessarily distinct), and $S_{0}\left(c_{j}\right) \neq 0$ for all $j=1, \ldots, \kappa$. Thus functions in $\mathbf{N}_{\kappa}$ and $\mathbf{S}_{\kappa}$ have meromorphic extensions to $\mathbf{C}_{+}$and $\mathbf{D}$. Boundary singularities of functions related as in (2.1) can be quite different: $S(z)=\exp (-(1+z) /(1-z))$ belongs to $\mathbf{S}_{0}$ and has values of modulus one on $\partial \mathbf{D}$ except for an essential singularity at $z=1$; its counterpart in $\mathbf{N}_{0}$ via $(2.1), f(z)=-\cot (z / 2)$, is real on the real axis except for simple poles at integer multiples of $2 \pi$.

\section{MAin Results}

First suppose that the given function is defined on the real line.

Theorem 3.1. Assume that $f(x)$ is a real-valued continuously differentiable function defined on a nonempty open subset $G$ of the real line. The following assertions are equivalent:

(i) there is a function $\hat{f} \in \mathbf{N}_{\kappa}$ which is holomorphic across $G$ and takes only real values on $G$ such that $\hat{f}(x)=f(x)$ for all $x$ in $G$;

(ii) the kernel (1.2) has $\kappa$ negative squares on $G \times G$.

For the unit circle, we have:

Theorem 3.2. Let $S(z)$ be a continuously differentiable function defined on a nonempty open subset $U$ of $\partial \mathbf{D}$ such that $|S(z)|=1$ for all $z \in U$. The following assertions are equivalent:

(i) there is a function $\hat{S} \in \mathbf{S}_{\kappa}$ which is holomorphic across $U$ and takes only values of modulus one on $U$ such that $\hat{S}(z)=S(z)$ for all $z$ in $U$;

(ii) the kernel (1.3) has $\kappa$ negative squares on $U \times U$. 
Vector-valued functions are used in the argument. Let $\mathbf{C}^{n}$ be $n$-dimensional complex Euclidean space, $\mathbf{C}^{m \times n}$ the set of $m \times n$ matrices with complex entries. The adjoint (conjugate transpose) of a matrix $A$ is written $A^{*}$. We call $A$ Hermitian if it is square and $A^{*}=A$. A signature matrix is an invertible square matrix $J$ such that $J=J^{*}=J^{-1}$.

Theorem 3.3 (Alpay-Dym [3]). Let $A, P \in \mathbf{C}^{n \times n}, C \in \mathbf{C}^{m \times n}$, and $J \in \mathbf{C}^{m \times m}$. Assume that $P$ is Hermitian and invertible, $J$ is a signature matrix, and

$$
P-A^{*} P A=C^{*} J C \text {. }
$$

Assume also that the only $\xi \in \mathbf{C}^{n}$ with $C(1-z A)^{-1} \xi \equiv 0$ is $\xi=0$. Let $\mathfrak{K}$ be the linear space of $\mathbf{C}^{m}$-valued functions of the form $f(z)=C(1-z A)^{-1} \xi, z \in \Omega$, where $\xi \in \mathbf{C}^{n}$ and $\Omega$ is the set of points $z$ such that $1-z A$ is invertible. Then $\mathfrak{K}$ is a finite-dimensional Krĕ̌n space in the inner product such that

$$
\langle f(\cdot), f(\cdot)\rangle_{\mathfrak{K}}=\xi^{*} P \xi
$$

whenever $f(z)=C(1-z A)^{-1} \xi$. The negative index of $\mathfrak{K}$ is equal to the number of negative eigenvalues of $P$, and $\mathfrak{K}$ has reproducing kernel of the form

$$
K(w, z)=\frac{J-\Theta(z) J \Theta(w)^{*}}{1-z \bar{w}},
$$

where $\Theta(z)$ is a holomorphic function on $\Omega$ with values in $\mathbf{C}^{m \times m}$. We can choose

$$
\Theta(z)=J-(1-z \bar{u}) C(1-z A)^{-1} P^{-1}\left(1-\bar{u} A^{*}\right)^{-1} C^{*}, \quad z \in \Omega,
$$

for any fixed point $u \in \partial D \cap \Omega$.

The space $\mathfrak{K}$ in Theorem 3.3 is denoted $\mathfrak{H}(\Theta ; \Omega)$. The last property of the pair $(C, A)$ in the hypotheses is called observability in linear system theory. We include the proof for the convenience of the reader. The meaning of the term "reproducing kernel" in this context can be seen in the proof; see also [2, Chapter 1].

Proof. The invertibility of $P$ and the fact that $\xi \rightarrow C(1-z A)^{-1} \xi$ is a one-to-

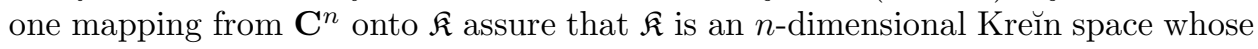
negative index is equal to the number of negative eigenvalues of $P$. By the definition of the inner product in $\mathfrak{K}$, the function

$$
K(w, z)=C(1-z A)^{-1} P^{-1}\left(1-\bar{w} A^{*}\right)^{-1} C^{*}
$$

has the properties that define a reproducing kernel: for every point $w \in \Omega$ and vector $\eta \in \mathbf{C}^{m}, K(w, \cdot) \eta$ belongs to $\mathfrak{K}$, and

$$
\langle f(\cdot), K(w, \cdot) \eta\rangle_{\mathfrak{K}}=\langle f(w), \eta\rangle_{\mathbf{C}^{m}}
$$

for every $f(\cdot)$ in $\mathfrak{K}$. A reproducing kernel is unique. We show that (3.2) holds if $\Theta(z)$ is defined by (3.3). By (3.1),

$$
\begin{aligned}
J-\Theta(z) J \Theta(w)^{*}=(1-u \bar{w}) C(1-u A)^{-1} P^{-1}\left(1-\bar{w} A^{*}\right)^{-1} C^{*} \\
+(1-z \bar{u}) C(1-z A)^{-1} P^{-1}\left(1-\bar{u} A^{*}\right)^{-1} C^{*} \\
-(1-z \bar{u})(1-u \bar{w}) C(1-z A)^{-1} P^{-1}\left(1-\bar{u} A^{*}\right)^{-1} \\
\cdot C^{*} J C(1-u A)^{-1} P^{-1}\left(1-\bar{w} A^{*}\right)^{-1} C^{*}
\end{aligned}
$$




$$
\begin{aligned}
& =C(1-z A)^{-1} P^{-1}\left(1-\bar{u} A^{*}\right)^{-1} \\
& \cdot\left[(1-u \bar{w})\left(1-\bar{u} A^{*}\right) P(1-z A)\right. \\
& +(1-z \bar{u})\left(1-\bar{w} A^{*}\right) P(1-u A) \\
& \left.-(1-z \bar{u})(1-u \bar{w})\left(P-A^{*} P A\right)\right] \\
& \cdot(1-u A)^{-1} P^{-1}\left(1-\bar{w} A^{*}\right)^{-1} C^{*} \\
& =C(1-z A)^{-1} P^{-1}\left(1-\bar{u} A^{*}\right)^{-1} \\
& \cdot(1-z \bar{w})\left(1-\bar{u} A^{*}\right) P(1-u A) \\
& \cdot(1-u A)^{-1} P^{-1}\left(1-\bar{w} A^{*}\right)^{-1} C^{*} \\
& =(1-z \bar{w}) K(w, z) \text {, }
\end{aligned}
$$

as required.

Lemma 3.4. In Theorem 3.3 suppose that $m=2$ and

$$
\Theta(z)=\left(\begin{array}{ll}
A(z) & B(z) \\
C(z) & D(z)
\end{array}\right)_{2 \times 2} .
$$

Then if $z \in \partial \mathbf{D} \cap \Omega$ and $|\gamma|=1,|C(z)|<|A(z)|,|C(z)|<|D(z)|,|B(z)-\gamma D(z)|=$ $|A(z)-\gamma C(z)|$, and $|A(z) \gamma+B(z)|=|C(z) \gamma+D(z)|$.

Proof. The form of the kernel (3.2) implies that $\Theta(z) J \Theta(z)^{*}=J$ and hence also $\Theta(z)^{*} J \Theta(z)=J$ for all $z \in \partial \mathbf{D} \cap \Omega$. These identities yield

$$
\begin{gathered}
|A(z)|^{2}-|B(z)|^{2}=1, \quad|D(z)|^{2}-|C(z)|^{2}=1, \\
A(z) \overline{C(z)}=B(z) \overline{D(z)}
\end{gathered}
$$

and

$$
\begin{gathered}
|A(z)|^{2}-|C(z)|^{2}=1, \quad|D(z)|^{2}-|B(z)|^{2}=1, \\
A(z) \overline{B(z)}=C(z) \overline{D(z)},
\end{gathered}
$$

for all $z \in \partial \mathbf{D} \cap \Omega$. The assertions follow by elementary manipulations from these relations.

We are now ready to prove the main results. It is sufficient to prove Theorem 3.2, since then Theorem 3.1 follows from transformations of the type (2.1)-(2.3).

Proof of Theorem 3.2. (i) $\Rightarrow$ (ii) Assume (i). Since $\hat{S}(z)$ belongs to $\mathbf{S}_{\kappa}$, the kernel

$$
K_{\hat{S}}(w, z)=\frac{1-\hat{S}(z) \overline{\hat{S}(w)}}{1-z \bar{w}}
$$

has $\kappa$ negative squares on a set $\Omega_{0} \times \Omega_{0}$, where $\Omega_{0}$ is the unit disk with at most finitely many points deleted. By $\left[2\right.$, Theorem 1.1.4], $K_{\hat{S}}(w, z)$ has $\kappa$ negative squares on $\Omega \times \Omega$ for some region $\Omega$ that contains $\Omega_{0} \cup U$. Let $\mathfrak{H}(\hat{S} ; \Omega)$ be the associated reproducing kernel Pontryagin space of holomorphic functions on $\Omega$. It has negative index $\kappa$. Since the restriction of $K_{\hat{S}}(w, z)$ to $U \times U$ is (1.3), restriction to $U$ is an isometry from $\mathfrak{H}(\hat{S} ; \Omega)$ onto a Pontryagin space $\mathfrak{H}(S ; U)$ which has reproducing kernel (1.3) and also has negative index $\kappa$. Hence (1.3) has $\kappa$ negative squares, which proves (ii). 
(ii) $\Rightarrow$ (i) As shown in $\S 1$, the result is known when $\kappa=0$. We shall obtain the general result by a reduction to this case.

Assume (ii). The construction depends on the choice of points $w_{1}, \ldots, w_{n}$ in $U$ such that the matrix $\left(K_{S}\left(w_{j}, w_{i}\right)\right)_{i, j=1}^{n}$ is invertible and has $\kappa$ negative eigenvalues. Such points exist by Lemma 2.1. They are distinct, since otherwise two rows in the matrix coincide and the matrix is not invertible. Put

$$
U_{0}=U \backslash\left\{w_{1}, \ldots, w_{n}\right\} .
$$

The restriction of the kernel (1.3) to $U_{0} \times U_{0}$ has $\kappa$ negative squares because the number of negative squares cannot exceed $\kappa$ by (ii), and for $w_{1}^{\prime}, \ldots, w_{n}^{\prime}$ sufficiently near $w_{1}, \ldots, w_{n}$, the matrix $\left(K_{S}\left(w_{j}^{\prime}, w_{i}^{\prime}\right)\right)_{i, j=1}^{n}$ has $\kappa$ negative eigenvalues by the continuity of (1.3) and the continuity of eigenvalues as functions of matrix entries $[8$, p. 540]. Let $\mathfrak{H}\left(S ; U_{0}\right)$ be the Pontryagin space of functions on $U_{0}$ with reproducing kernel (1.3) restricted to $U_{0} \times U_{0}$. The negative index of $\mathfrak{H}\left(S ; U_{0}\right)$ is $\kappa$. It is enough to show that there exists a function $\hat{S} \in \mathbf{S}_{\kappa}$ which is holomorphic across $U_{0}$ and takes only values of modulus one on $U_{0}$ such that $\hat{S}(z)=S(z)$ for all $z$ in $U_{0}$. To include the omitted points $w_{1}, \ldots, w_{n}$, we then only need to apply the conclusion beginning with a different choice of points $w_{1}, \ldots, w_{n}$ in $U$.

The hypotheses of Theorem 3.3 are satisfied with

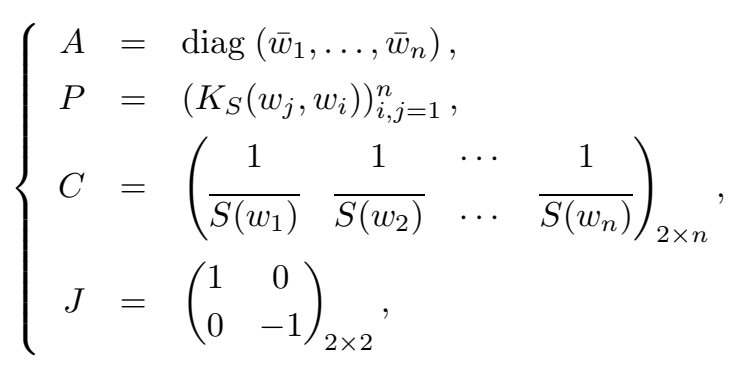

and $m=2$. The function $\Theta(z)$ produced by Theorem 3.3 is defined on $\Omega=$ $\mathbf{C} \backslash\left\{w_{1}, \ldots, w_{n}\right\}$. The space $\mathfrak{H}(\Theta ; \Omega)$ has negative index $\kappa$ and is spanned by the $\mathbf{C}^{2}$-valued functions

$$
h_{j}(z)=\left(\frac{1}{S\left(w_{j}\right)}\right)_{2 \times 1} \frac{1}{1-z \bar{w}_{j}}, \quad j=1, \ldots, n,
$$

with inner product such that

$$
\left\langle h_{j}(\cdot), h_{i}(\cdot)\right\rangle_{\mathfrak{H}(\Theta, \Omega)}=K_{S}\left(w_{j}, w_{i}\right), \quad i, j=1, \ldots, n .
$$

Since $m=2, \Theta(z)$ has the form (3.4).

There is a natural isometry $\Phi$ from $\mathfrak{H}(\Theta ; \Omega)$ into $\mathfrak{H}\left(S ; U_{0}\right)$, namely, multiplication by the matrix $(1-S(z))=(1-S(z))_{1 \times 2}$ followed by restriction to $U_{0}$. In fact, every element of $\mathfrak{H}(\Theta ; \Omega)$ has the form $h(z)=\sum_{j=1}^{n} c_{j} h_{j}(z)$ for some numbers $c_{1}, \ldots, c_{n}$, and

$$
\langle h(\cdot), h(\cdot)\rangle_{\mathfrak{H}(\Theta ; \Omega)}=\sum_{i, j=1}^{n} \bar{c}_{i} K_{S}\left(w_{j}, w_{i}\right) c_{j}
$$


by (3.6). Then $(1-S(z)) h(z)=\sum_{j=1}^{n} K_{S}\left(w_{j}, z\right) c_{j}$ belongs to $\mathfrak{H}\left(S ; U_{0}\right)$ as a function of $z \in U_{0}$, and

$$
\langle(1-S(\cdot)) h(\cdot),(1-S(\cdot)) h(\cdot)\rangle_{\mathfrak{H}\left(S ; U_{0}\right)}=\sum_{i, j=1}^{n} \bar{c}_{i} K_{S}\left(w_{j}, w_{i}\right) c_{j}
$$

by the definition of a reproducing kernel. Thus $\Phi$ is an isometry which maps $\mathfrak{H}(\Theta ; \Omega)$ into $\mathfrak{H}\left(S ; U_{0}\right)$. Its range is a regular subspace $\mathfrak{L}$ of $\mathfrak{H}\left(S ; U_{0}\right)$. The idea of a mapping of this type appears in a different form in [5].

For all $w, z \in U_{0}$,

$$
\begin{aligned}
& \frac{1-S(z) \overline{S(w)}}{1-z \bar{w}}=(1-S(z)) \frac{J-\Theta(z) J \Theta(w)^{*}}{1-z \bar{w}}(1-S(w))^{*} \\
& +(1-S(z)) \frac{\Theta(z) J \Theta(w)^{*}}{1-z \bar{w}}(1-S(w))^{*} .
\end{aligned}
$$

The first term on the right side of (3.7) is the reproducing kernel for $\mathfrak{L}$. Hence the second term is the reproducing kernel for $\mathfrak{M}=\mathfrak{H}\left(S ; U_{0}\right) \ominus \mathfrak{L}$. Since $\mathfrak{H}\left(S ; U_{0}\right)$ and $\mathfrak{L}$ each have negative index $\kappa, \mathfrak{M}$ is a Hilbert space. Therefore the second term on the right side of (3.7) is a nonnegative kernel. In expanded form, it is given by

$$
\begin{aligned}
(1-S(z)) \frac{\Theta(z) J \Theta(w)^{*}}{1-z \bar{w}}\left(\begin{array}{ll}
1 & -S(w))^{*}
\end{array}\right. & (1-z \bar{w})^{-1}\{[A(z)-S(z) C(z)][\overline{A(w)}-\overline{S(w)} \overline{C(w)}] \\
& \quad-[B(z)-S(z) D(z)][\overline{B(w)}-\overline{S(w)} \overline{D(w)}]\} \\
= & {[A(z)-S(z) C(z)][\overline{A(w)}-\overline{S(w)} \overline{C(w)}] \frac{1-S_{0}(z) \overline{S_{0}(w)}}{1-z \bar{w}}, }
\end{aligned}
$$

where

$$
S_{0}(z)=\frac{-B(z)+S(z) D(z)}{A(z)-S(z) C(z)} .
$$

By Lemma 3.4, $S_{0}(z)$ is well defined on $U_{0}$. It is continuously differentiable, has values of unit modulus, and satisfies (ii) with $\kappa=0$. Since the result is known in this case, there is a function $\hat{S}_{0}(z)$ in $\mathbf{S}_{0}$ which is holomorphic across $U_{0}$ whose restriction to $U_{0}$ is $S_{0}(z)$. The function

$$
\hat{S}(z)=\frac{A(z) \hat{S}_{0}(z)+B(z)}{C(z) \hat{S}_{0}(z)+D(z)}
$$

is well defined and holomorphic on a neighborhood $W$ of $U_{0}$ by Lemma 3.4, and its restriction to $U_{0}$ is $S(z)$. It remains to show that $\hat{S} \in \mathbf{S}_{\kappa}$. To do this, we reverse the preceding calculations with $S(z)$ and $S_{0}(z)$ replaced by $\hat{S}(z)$ and $\hat{S}_{0}(z)$. We find that

$$
\begin{aligned}
\frac{1-\hat{S}(z) \overline{\hat{S}(w)}}{1-z \bar{w}}= & (1-\hat{S}(z)) \frac{J-\Theta(z) J \Theta(w)^{*}}{1-z \bar{w}}(1-\hat{S}(w))^{*} \\
& +[A(z)-\hat{S}(z) C(z)][\overline{A(w)}-\overline{\hat{S}(w)} \overline{C(w)}] \frac{1-\hat{S}_{0}(z) \overline{\hat{S}_{0}(w)}}{1-z \bar{w}}
\end{aligned}
$$

for all $w, z$ in $W$. The first term on the right side of (3.9) has $\kappa$ negative squares and the second is nonnegative on $W \times W$. By [2, Theorem 1.5.5], the left side of 
(3.9) has at most $\kappa$ negative squares on $W \times W$. Its restriction to $U_{0} \times U_{0}$ already has $\kappa$ negative squares. It follows that $\hat{S} \in \mathbf{S}_{\kappa}$, and we are done.

The methods of this paper can be adapted to operator-valued functions. The authors hope to take up this extension and related questions in interpolation theory in future work.

\section{REFERENCES}

1. D. Alpay, A. Dijksma, J. Rovnyak, and H. S. V. de Snoo, Reproducing kernel Pontryagin spaces, Holomorphic Spaces (S. Axler, J. McCarthy, and D. Sarason, eds.), MSRI Publications, vol. 33, Cambridge University Press, New York, 1998, pp. 425-444.

2. - Schur functions, operator colligations, and reproducing kernel Pontryagin spaces, Oper. Theory: Adv. Appl., Vol. 96, Birkhäuser, Basel, 1997. CMP 97:17

3. D. Alpay and H. Dym, On a new class of reproducing kernel spaces and a new generalization of the Iohvidov laws, Linear Algebra Appl. 178 (1993), 109-183. MR 94g:46034

4. N. Aronszajn, Theory of reproducing kernels, Trans. Amer. Math. Soc. 68 (1950), 337-404. MR 14:479c

5. L. de Branges and J. Rovnyak, Canonical models in quantum scattering theory, Perturbation Theory and its Applications in Quantum Mechanics (Proc. Adv. Sem. Math. Res. Center, U.S. Army, Theoret. Chem. Inst., Univ. of Wisconsin, Madison, Wis., 1965), Wiley, New York, 1966, pp. 295-392. MR 39:6109

6. L. E. Dickson, On the rank of a symmetrical matrix, Annals of Math. 15 (1913), 27-28.

7. W. F. Donoghue, Jr., Another extension of Loewner's theorem, J. Math. Anal. Appl. 110 (1985), 323-326. MR 87b:30058

8. R. A. Horn and C. R. Johnson, Matrix analysis, Cambridge University Press, Cambridge, 1985. MR 87e: 15001

9. A. Korányi, On a theorem of Löwner and its connections with resolvents of selfadjoint transformations, Acta Sci. Math. Szeged 17 (1956), 63-70. MR 18:588c

10. M. G. KreĬn and H. Langer, Über die verallgemeinerten Resolventen und die charakteristische Funktion eines isometrischen Operators im Raume $\Pi_{\kappa}$, Hilbert space operators and operator algebras (Proc. Internat. Conf., Tihany, 1970), North-Holland, Amsterdam, 1972, pp. 353-399. Colloq. Math. Soc. János Bolyai, 5. MR 54:11103

11. K. Löwner, Über monotone Matrixfunktionen, Math. Z. 38 (1934), 177-216.

12. M. Rosenblum and J. Rovnyak, Restrictions of analytic functions. I, Proc. Amer. Math. Soc. 48 (1975), 113-119. MR 53:3764a

13. _ Hardy classes and operator theory, Oxford Mathematical Monographs, Oxford University Press, New York, 1985, Dover reprint, 1997. MR 87e:47001; MR 97j:47002

14. L. Schwartz, Sous-espaces hilbertiens d'espaces vectoriels topologiques et noyaux associés (noyaux reproduisants), J. Analyse Math. 13 (1964), 115-256. MR 31:3835

15. P. Sorjonen, Pontrjaginräume mit einem reproduzierenden Kern, Ann. Acad. Sci. Fenn. Ser. A I Math. (1975), no. 594, 30 pp. MR 53:8875

Department of Mathematics, Ben-Gurion University of the Negev, P. O. Box 653, 84105 BeER-SheVA, ISRAel

E-mail address: dany@math.bgu.ac.il

Department of Mathematics, University of Virginia, Charlottesville, Virginia 229033199

E-mail address: rovnyak@irginia.EDU 